\title{
Visualizing the Optically Induced Near-fields of Nanoplasmonics with Ultrafast Transmission Electron Microscopy
}

\author{
Aycan Yurtsever ${ }^{1}$ \\ ${ }^{1}$ Centre Energie, Matériaux et Télécommunications, Institut National de la Recherche Scientifique \\ (INRS), 1650 Boulevard Lionel-Boulet, Varennes, QC, J3X 1S2, Canada
}

Localized electric fields that are induced optically exhibit unique phenomena of fundamental importance to nanoplasmonics. In recent years, they have been considered for efficient photovoltaic and light harvesting devices, single molecule detection, biomolecular labeling and manipulation, and surface enhanced Raman scattering [1]. Several methodologies have been utilized for probing the near-fields, and these include optical methods, cathodoluminesce and transmission electron microscopy [2]. The latter probes the fields with sub-nanometer precision but the measured quantity is the dielectric response of the system to an impinging swift electron, and not to a photon excitation.

Ultrafast transmission electron microscopy (UTEM) offers unique approaches for studying photon induced near-fields by combining the versatility of transmission electron microscopes (TEM) and optical techniques. In this methodology, sample is excited by a femtosecond (fs) laser pulse and imaging is performed with ultra-short electron packets that are extracted from the cathode via the photoelectric effect. This imaging, and excitation, approach is in contrast to conventional TEM where continuous beam of electrons are used. The plasmonic visualization method presented here is based on an inelastic photon-electron interaction process, where the probing electrons gain, or lose, energy equal to the integer multiple of the photon quanta $(2.4 \mathrm{eV})$. These electrons are then analyzed with a spectrometer, in a fashion similar to energy-filtered TEM.

Plasmonic fields of optically excited silver nanoparticles are shown in Fig. 1 for two different linear polarizations of the excitation laser. All particles show a dipolar behavior, as expected at the Rayleigh limit. With such high-field-of-view images one can simultaneously visualize the plasmonic fields of the whole ensemble and the effect of particle-particle and particle-substrate interactions on these fields [3]. For instance, on the plasmon images, it is evident that not all dipoles are perfectly aligned with the linear polarization. Also, step edges on the layered-graphene substrate an $\mathrm{d}$ their effect on the dipolar fields can be observed.

When two particles are brought in close proximity to each other, comparable or closer than their evanescent decay length of $\lambda / 2 \pi$, their near-fields start to interact coherently. The effect of this interaction on the near-field image is shown in Fig. 2. Void-channels open up between the particles, where the longitudinal component $\left(E_{z}\right)$ of the electric field vanishes. The shape and width of this unique feature depend on the particle separation and polarization. In the corresponding bright-field images, there is nothing in the space between the particles, yet, in the plasmon images high contrast is observed in the in-between space. Similar channels also exist for a nanoparticle chain [4].

UTEM provides unique capabilities to approach nanoplasmonics. Here, we have demonstrated the visualization of a particle ensemble with high throughput of plasmonic information. Existence of narrow channels in the fields of particle dimers and chains were also observed. These advances were made possible by the optical excitation and resolutions available to our methodology [5]. 


\section{References}

[1] S.A. Maier, "Plasmonics: Fundamentals and applications", Springer, New York (2007)

[2] F.J. García de Abajo, Reviews of Modern Physics, 82, 209-275 (2010)

[3] A. Yurtsever and A. H. Zewail, Nano Letters, 12, 3334 (2012)

[4] A. Yurtsever, J. S. Baskin and A. H. Zewail, Nano Letters 12, 5027 (2012)

[5] Experiments were conducted in the group of Prof. Ahmed Zewail at the California Institute of Technology. The original work was published in [3] and [4].

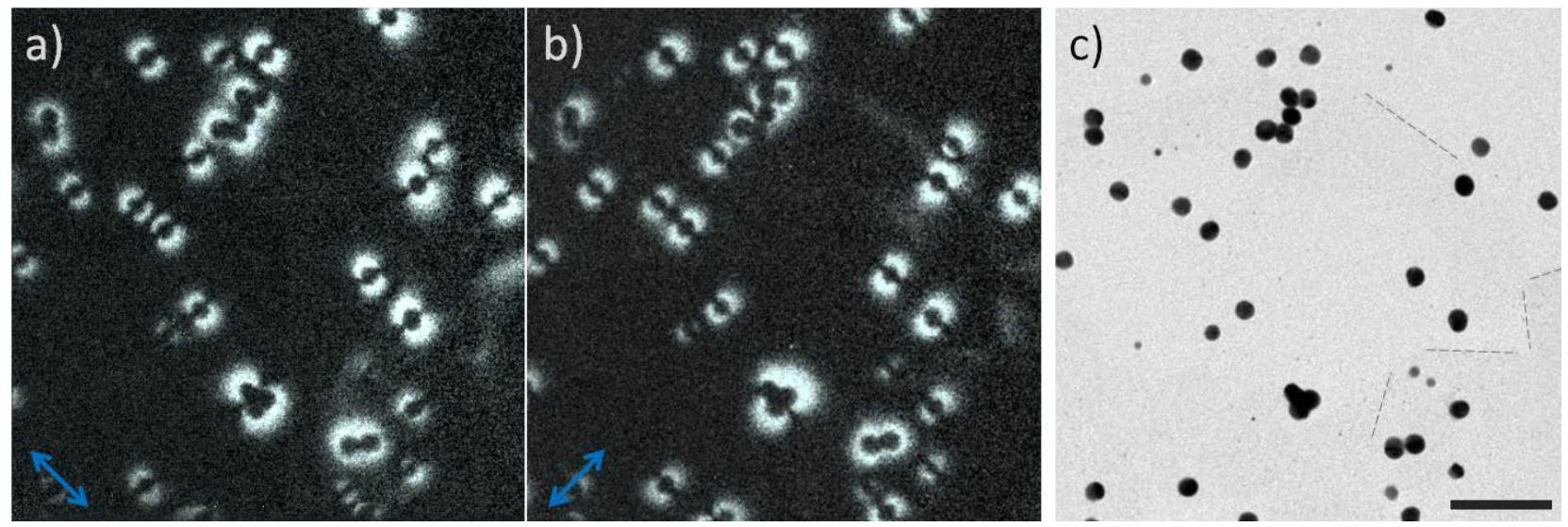

FIG. $1 \mathrm{a} \& \mathrm{~b})$ Localized near-fields of a nanoparticle ensemble for two linear polarizations. Particles exhibit dipolar behavior for each polarization of the incident laser; polarization is shown with arrows at the bottom-left. The wavelength of the laser excitation is $520 \mathrm{~nm}(\lambda)$, its pulse duration is $220 \mathrm{fs}$ and peak fluence is $4 \mathrm{~mJ} / \mathrm{cm}^{2}$; the average energy of the probing electron packets is $200 \mathrm{keV}$. Images are taken at a stroboscopic fashion at $400 \mathrm{kHz}$ repetition rate. c) Bright-field image of the ensemble. Particles are silver and substrate is layered-graphene. Dashed lines de note step edges on the substrate that can be identified by change of contrast in the bright-field image; scale bar is $500 \mathrm{~nm}$.
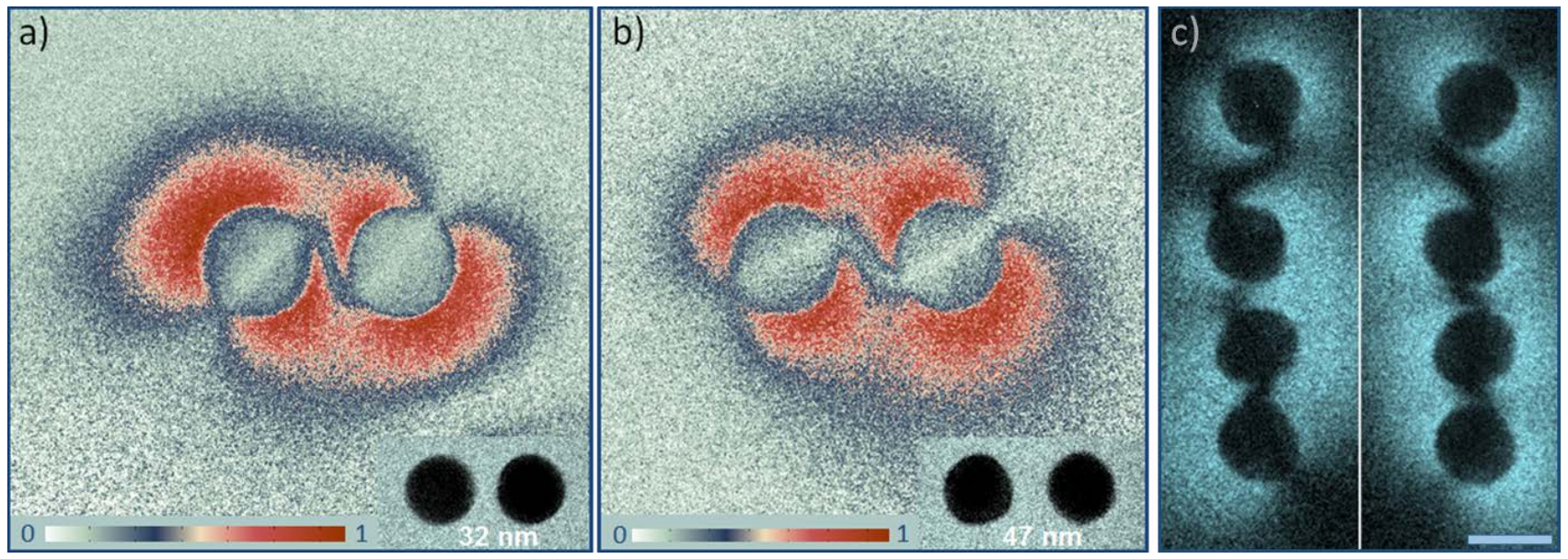

FIG. $2 \mathrm{a} \& \mathrm{~b}$ ) Near-fields of two nanoparticle dimers with an edge-to-edge distance of $32 \mathrm{~nm}$ (a) and 47 $\mathrm{nm}$ (b) with false-color mapping. Bright-field images of the dimers are shown in the inset at the bottom right; plasmon images are displayed at a higher magnification than the bright-field images. In the falsecolor bars, white indicates the lowest intensity and red the highest. Polarization of the laser field is at $45^{\circ}$ (counterclockwise) from the vertical. c) Near-fields and their channels for a particle chain; polarizations are $+60^{\circ}$ and $-60^{\circ}$ from the vertical and the scale bar is $100 \mathrm{~nm}$. 